\title{
Rett Syndrome: a challenge to an anaesthetist
}

\author{
Namrata Maheshwari ${ }^{1}$, Deepak Gandhi \\ *Assistant Professor ${ }^{l}$, Dept of Anaesthesiology, Sir Grant Medical College and JJ Hospital, Mumbai ${ }^{2}$.
}

*Corresponding author:drnamratamaheshwari@gmail.com

Key words: Rett's Syndrome, regional anaesthesia, scoliosis

\begin{abstract}
Rett syndrome is a progressive neurological disorder of females characterized by dementia, severe autism, movement disorders, growth retardation, microcephaly, seizures, muscle wasting, scoliosis and an abnormality of respiratory control ${ }^{1}$. These patients may show a characteristic abnormal respiratory pattern consisting of episodes of tachypnea and irregular respiration, intermixed with apnoea, observed only during wakefulness. ${ }^{2}$ Anaesthesia is challenging in this rare subgroup of patients with its multiple comorbid presentations. This report provides a practical approach comprising of vigilant monitoring and proactive actions like preoperative oxygenation, anti-epileptic medications, adequate fluid infusion and unilateral spinal anaesthesia restricting level to $\mathrm{T} 10$ to avoid respiratory distress for anaesthesia in a patient with Rett syndrome for sub trochanteric fracture nailing.
\end{abstract}

A 21 year old female weighing 30kgs, a diagnosed case of Rett syndrome, born of a non consanguineous marriage, came with a history of fall with subtrochanteric fracture of the right femur. Surgery was planned for her in the form of femur interlocking with nailing.

Patient gets generalised tonic clonic convulsions and is on oral carbamazepine since birth. She had neuromuscular scoliosis secondary to Rett's syndrome, which was diagnosed at the age of five with a hump in the thoracic region and the lumbar spine deviated away from the midline. She also was diagnosed with post polio residual paralysis of right lower limb.

She was preterm delivered as a normal vaginal delivery at 32 weeks with a birth weight of 2.5 kgs. She cried a little late after birth. Her developmental milestones were normal till 1 year of age, when she stopped meeting developmental guidelines and was diagnosed as a case of Rett syndrome at the age of 3 . She was also diagnosed to have bladder and bowel incontinence.

On examination, she was cachetic, with poor nutrition, not oriented to time, place and person. She was having abnormal tremors with hand wringing. She was unable to speak and was totally dependent in all aspects of daily care. Her head circumference was less than the third percentile.

Mallampati classification could not be judged. Mouth hygiene was poor with excessive oral secretions.

Respiratory system- air entry was bilaterally diminished in the bases with rhonchi and conducted sounds. Pulmonary function test was desirable due to scoliosis, but it was not possible due to lack of cooperation.

Cardiovascular system- was normal.

Her blood haemogram and biochemistry were normal. Electrocardiogram was also unremarkable.

$\mathrm{X}$ ray chest showed increased bronchovascular markings with deviation of the trachea.

\section{Anaesthetic management}

Anaesthesia was a challenge in this patient as regional anaesthesia would be difficult due to scoliosis in thoraco-lumbar region. General anaesthesia with endotracheal intubation was not the choice as these patients have scoliosis, with poor respiratory reserve, and high chances of desaturation. 
After informed consent from parents and risk stratification, patient was taken into the operation theatre and monitored with electrocardiogram, pulse oximetry, non invasive blood pressure and temperature probe. 20 gauge intravenous cannula was established on the left arm and i.v. Ringer lactate solution was started.

Vital parameters were heart rate-98/min, oxygen saturation on air $92 \%$ (100\% on oxygen), and blood pressure-110/78mmHg. Unilateral spinal anaesthesia was planned. After all aseptic precautions, patient was positioned in the left lateral position.

The lumbar spine was very difficult to palpate as patient was having thoracolumbar scoliosis with a hump in the lower thoracic region and the lumbar spine was shifted away from the midline. As patient was not co-operative $0.2 \mathrm{mg}$ i.v. glycopyrrolate, $4 \mathrm{mg}$ i.v. ondansetron and $25 \mathrm{mg}$ i.v. ketamine were given before the lumbar puncture. After three attempts the dural puncture was successful with a $25 \mathrm{~g}$ spinal needle and $0.5 \%$ bupivacaine $2 \mathrm{cc}$ along with $25 \mathrm{mcg}$ fentanyl were given slowly. The patient was placed in the right lateral position, as the surgery was on right limb. The spinal level was assessed by using a behavioural pain score (graded using facial expression, crying, and movements) because of the patient's inability to communicate.

Analgesic level to $10^{\text {th }}$ thoracic dermatome (T-10) was achieved on right side. Intra-operatively the patient was given 4 lit/min $\mathrm{O}_{2}$ through face mask and all vital parameters were stable including $\mathrm{SpO}^{2}(100 \%)$. During this one and a half hour of surgical procedure she was given $500 \mathrm{ml}$ of Ringer lactate and $500 \mathrm{ml}$ of $5 \%$ dextrose in $0.45 \%$ normal saline.

High spinal was avoided by giving low drug dosage and placing the patient head high with pillow under the shoulder. Blood loss during the procedure was about $200 \mathrm{ml}$. She was maintaining a urine output of around $50 \mathrm{ml} /$ hour.

Post operatively her pulse was $88 / \mathrm{min}$, blood pressure of $110 / 70 \mathrm{~mm} \mathrm{Hg}$ and $\mathrm{SpO}^{2}$ was $100 \%$ on $2 \mathrm{lit} / \mathrm{min}$ oxygen. She was awake, responding well and did not require any further sedation and analgesia. Her oxygen saturation was $94 \%$ on 1 st post-operative day on room air with no respiratory distress. She was discharged on $7^{\text {th }}$ post-operative day and had an uneventful recovery.

\section{Discussion}

Rett in 1966 described a syndrome of progressive deterioration of higher brain functions occurring only in girls ${ }^{3}$. The syndrome is characterised by a period of normal development for the period of 4-18 months followed by rapid developmental regression leading to dementia with autistic behaviour, axial hypotonia, hand wringing, hand washing movements and limb spasticity as seen in our patient. Various movement disorders are also associated with this syndrome including choreoathetosis, dystonia, myoclonic jerks and stereotypic automatism ${ }^{\mathbf{4 , 5}}$. Our patient was having generalised tonic clonic convulsions with myoclonic jerks. Neurological changes like psychomotor regression, social withdrawal, loss of speech and babbling develops gradually, which was also seen in our case.

There are reports of irregular breathing and apnoea when patient is awake, but no such irregularities occur during sleep when breathing is stable ${ }^{6}$. Our patient though did not show any such sleep variations.

Scoliosis with a double curved deformity is characteristic and is found in $80 \%$ of all the reported cases along with significant anatomic and physiologic problems related to scoliosis.

Concerns for anaesthetist are lack of cooperation, muscle wasting, abnormal continuous limb movements, abnormal respiratory control, difficult positioning secondary to scoliosis and chest deformity, vasomotor instability, and altered sensitivity to pain. These patients have a high pain threshold ${ }^{7}$, which may be due to reduced sensitivity to pain resulting from increased $\beta$-endorphin concentrations in the cerebrospinal fluid or impaired abnormal neural transmission and pain processing $\mathbf{8 9}$.

Other features of interest to anaesthetist are air swallowing, screaming spells, vasomotor hyper reactivity of feet, breath holding, episodic hyperventilation, altered sensitivity to painful stimuli and neurogenic scoliosis. 
These children may be at risk of recurrent aspiration and impaired respiratory function from muscle weakness and thoracic deformity.

They may show a characteristic abnormal respiratory pattern consisting of episodes of tachypnoea and irregular respiration, intermixed with apnoea, observed only during wakefulness. These respiratory effects might confound the respiratory side effects of opiod analgesics.

Frequent desaturation may cause permanent hypoxic damage and may lead to cerebral deterioration.

Metabolic abnormalities in the form of elevated ammonia levels have been reported in Rett's syndrome but are not seen as a consistent feature of this condition.

Rett syndrome although has been described almost 45 yrs ago, awareness of this syndrome is very recent. There is no evidence to clarify the cause of this disorder and the diagnosis is made by exclusion.

Our patient exhibited the major features of Rett syndrome with developmental delay, scoliosis, and loss of speech.

We were able to give spinal anaesthesia to our patient with difficulty. Vigilant monitoring and proactive actions which included preoperative oxygenation, anti-epileptic medications, adequate fluid infusion and restricting spinal level to $10^{\text {th }}$ thoracic dermatome (T-10) to avoid respiratory distress, she had an uneventful intra and post operative period with no signs of apnoea and desaturation.

\section{References:}

1. Armstrong DD. Review of Rett syndrome. J Neuropathol Exp Neurol 1997; 56:843-9.

2. Kerr AM. A review of the respiratory disorder in the Retts syndrome. Brain Dev 1992; 14(Suppl):S43-5.

3. Rett-A. Ueber eom eogemartiges hiranatropisches syndrome bei Hyperammoniae im kindersalter.Weiner Med Wochenschrift 1966;116;723-38.

4. AI-Mateen M, Phillppart M, Shields WD. Retts Syndrome: a commonly overlooked progressive encephalopathy in girls. Am J Dis Child 1986; 140:761-65.

5. Hagberg B, Aicardi J, Dias K, Ramoso. A progressive syndrome of autism, dementia, ataxia and loss of purposeful hand use in girls: Retts Syndrome: Report of 35 cases; Ann.Neurol 1984;14;471-79.

6. Cirignotta F, Lugaresi E.Montagna P. Breathing impairment in Retts syndrome. Am J.Med Genet 1986; 24.(Suppl I):167-73.

7. Coleman M, Brubaker J, Hunter K, Smith G. Rett syndrome: a survey of North American patients. $J$ Mental Deficiency Res 1988; 32:117-24.

8. Brase DA, Myer EC, Dewey WL. Possible hyperendorphinergic pathophysiology of the Rett syndrome. Life Sci 1989; 45:359 - 66.

9. Myer EC, Tripathi HL, Brase DA, et al. Elevated CSF beta endorphin immunoreactivity in Rett syndrome: report of 158 cases and comparison with leukaemic children. Neurology 1992; 42:357- 60 . 